\title{
Correction to: Simplified therapeutic guidelines: the main tool of antimicrobial stewardship programs associated with optimal antibiotic therapy
}

\author{
Pierre-Marie Roger ${ }^{1,2,3}$. Anne Espinet ${ }^{4} \cdot$ Delphine Ravily $^{5}$ - Marie-José Meyer ${ }^{6}$ Francine Moll ${ }^{7}$ Eve Montera ${ }^{8}$. \\ Agnès Rancezot $^{9} \cdot$ Véronique Dautezac $^{10} \cdot$ Olivier Pantaloni $^{11}$
}

Published online: 29 January 2022

(c) Springer-Verlag GmbH Germany, part of Springer Nature 2022

\section{Correction to: European Journal of Clinical Microbiology \& Infectious Diseases https://doi.org/10.1007/s10096-021-04317-z}

"V3" was incorrectly added at the end of the main title of the originally published article. The correct title should be:

Simplified therapeutic guidelines: the main tool of antimicrobial stewardship programs associated with optimal antibiotic therapy

The original paper has been corrected.

Publisher's note Springer Nature remains neutral with regard to jurisdictional claims in published maps and institutional affiliations.

The original article can be found online at https://doi.org/10.1007/ s10096-021-04317-z.

Pierre-Marie Roger roger@elsan.care

1 Infectiologie, Clinique Les Fleurs, 332 Ave Frédéric Mistral, 83110 Ollioules, France

2 Faculté de Médecine, Université Des Antilles, Campus de Fouillole, Pointe-à-Pitre, Guadeloupe, France

3 Clinique Les Fleurs, Ave Frédéric Mistral, 83190 Ollioules, France

4 Clinique St Augustin, 114, Ave d'Ares, 33074 Bordeaux, France

5 Clinique de l'Orangerie, 29, Allée de la Robertsau, 67000 Strasbourg, France

6 Hôpital Privé Guillaume de Varye, 210 Rte de Vouzeron, 18230 St Doulchard, France
7 Recherche Clinique, Clinique du Cap d'Or, 1361 Avenue des Anciens Combattants Français, 83500 La Seyne-sur-Mer, France

8 Clinique St Roch, Rue Ambroise Croizat, 66330 Cabestany, France

9 Clinique Médicale Et Cardiologique, Rue de Lourdes, 64320 Aressy, France

10 Clinique du Sidobre, Chemin de St Hippolyte, 81100 Castres, France

11 Clinique St Pierre, 169 Ave de Prades, 66000 Perpignan, France 